\title{
Les nouvelles formes galéniques et la voie sous-cutanée en focus : conséquences pour l'organisation des soins, pour la délivrance et pour le vécu du traitement
}

\author{
New Dosage Forms and a Focus on Subcutaneous Administration: Consequences for the Care \\ Structure, for its Delivery and for the Success of Treatment
}

\author{
G. Milano \\ (C) Springer-Verlag France 2014
}

Trois articles sont ici rassemblés afin de capter notre attention et nous amener à réfléchir sur une des avancées récentes du traitement du cancer, à savoir l'évolution des formes galéniques des médicaments anticancéreux. Le traitement oral va de pair avec l'introduction remarquée et remarquable des thérapies ciblées faisant appel aux inhibiteurs de tyrosinekinase, dans la plupart des cas. Cette simplicité d'administration particulièrement appréciée par le patient ne doit pas cacher la réalité d'un vrai médicament anticancéreux qui découvre la deuxième face de Janus, à savoir sa toxicité. L'article de Christelle Levy et Aurélie Bouhelier nous le rappelle et nous interpelle plus particulièrement sur la voie sous-cutanée (SC). Ce mode d'administration est peu utilisé en oncologie mais pourtant il percute déjà deux traitements majeurs : le très innovant denosumab et le poids lourd trastuzumab.

L'article sur les aspects biotechnologiques et les implications cliniques donne en synthèse des considérations très pratiques sur la voie SC comparée aux abords i.v. et i.m.
L'article s'attache aussi à décrire globalement les déterminants et les limites de l'absorption par voie SC. Plus précisément, un effet de loupe est appliqué à l'Herceptin ${ }^{\circledR}$ et son administration par voie SC. À travers la comparaison des administrations de l'Herceptin ${ }^{\circledR}$ par voies SC et i.v. les auteurs mettent l'accent sur les critères d'efficacité et de toxicité mais également la pharmacocinétique, outil qui révèle ici toute son utilité médicale.

Enfin, un dernier article, peut surprendre par son titre : « cancer j'aurai ta peau ». On comprend d'emblée qu'une autre dimension est accordée ici à la question posée par l'administration SC, c'est celle de la vision du psychiatre qui donne la parole au patient face à sa prise en charge et nous révèle chez ce dernier une singulière compétence à lutter contre sa maladie.

On se pique de le savoir...

Liens d'intérêts : G. Milano déclare être consultant pour le laboratoire Roche.

G. Milano $(\square)$

Laboratoire d'oncopharmacologie, centre Antoine-Lacassagne, F-06189 Nice, France

e-mail : gerard.milano@nice.unicancer.fr 